\title{
An Exciting Aspect of Nanotechnology: Unimolecular Electronics
}

\author{
R. M. Metzger ${ }^{1,2}$ \\ ${ }^{1}$ Laboratory for Molecular Electronics, Department of Chemistry, University of Alabama, Tuscaloosa, AL 35487, USA \\ ${ }^{2}$ Institute for Materials Science, Dresden University of Technology, D-01062 Dresden, Germany
}

\begin{abstract}
This is a brief update on our experimental work towards better one-molecule-thick monolayer rectifiers of electrical current, and on theoretical progress towards a one-molecule amplifier of electrical current. This program aims to provide electronic devices at the 2 to $3 \mathrm{~nm}$ level, as a dramatic advance towards practical integrated circuits of the future.
\end{abstract}

\section{Introduction}

The field of unimolecular electronics started with a 1974 paper by Arieh Aviram and Mark Ratner (AR) [1]. As reviewed elsewhere [2,3] eleven rectifiers have been completely characterized in our laboratory, and several others have been reported by others [4, 5]. For strictly one-molecule two-probe measurements, mechanical break junctions (MBJ) [6, 7], electromigration break junctions (EMBJ) [8], scanning tunnelling microscopy (STM) [9] and scanning probe break junctions (SPBJ) [10] permit the measurement of the electrical current through a single molecule that bridges, say, a $1 \mathrm{~nm}$ gap between electrodes. Alas, at present nobody can fabricate three nanoelectrode tips that are about $2 \mathrm{~nm}$ from each other (the challenge is getting insulating oxides between electrodes that are as thin as $2 \mathrm{~nm}$ ). Here we summarize older measurements, report on on-going work on several new candidate rectifier molecules, and discuss a recent theory on how a three-terminal molecule could act as an electrical power amplifier [11].

\section{Monolayer rectifiers}

AR proposed that a single-molecule rectifier (or diode) of the type $D-\sigma-A$, where $D$ is a strong electron donor moiety, A is a strong electron acceptor moiety, and $\sigma$ is a saturated (or twisted aromatic) covalent link between D and A. By definition, a D moiety has a relatively low ionization potential, while the A moiety has a relatively large electron affinity. The experimental verification of AR rectification involved at first Langmuir-Blodgett (LB) or Langmuir-Schaefer (LS) monolayers measured between macroscopic metal electrodes ( $\mathrm{Al}, \mathrm{Mg}, \mathrm{Pt}$ [12], and later $\mathrm{Au}$ ), then chemisorbed monolayers tapped by a macroscopic Au electrode [13], and finally MBJ [4] and scanning tunnelling microscopy [5] for single molecules. Fig. 1 shows the chemical structures of eleven rectifiers, and Table 1 shows their electrical characteristics.

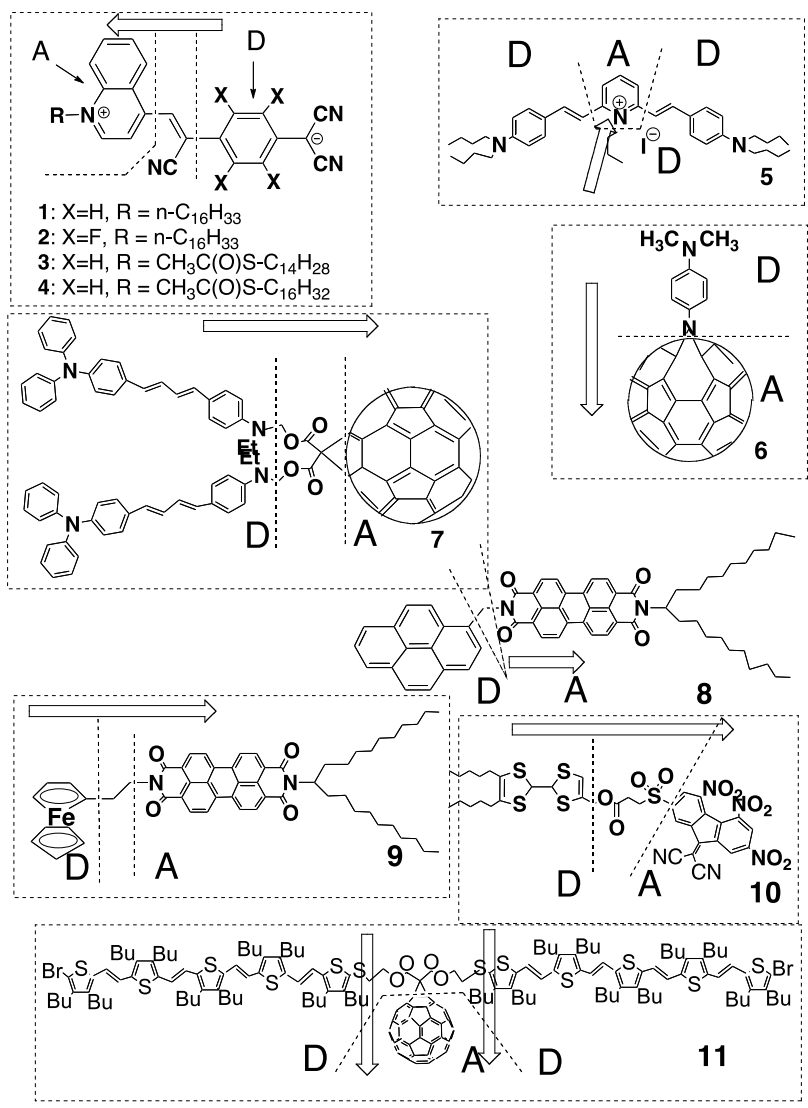

Fig. 1. Structures of the eleven rectifiers (1-11) characterized at the University of Alabama.

The AR rectification, or asymmetric conduction model, is ascribed to resonant transfer of electrons across the two metal/molecule junctions when the Fermi levels of the 
electrodes match the HOMO or LUMO energies of the molecule.

Table 1. Results for eleven monolayer rectifiers (structures in Fig. 1): IVT=maximum of intervalence transfer band; $\mathrm{RR}=$ rectification ratio at maximum measured voltage $\mathrm{V} \equiv-\mathrm{I}(\mathrm{V}) / \mathrm{I}(-\mathrm{V})$; "Survives cycling?" $\equiv$ if "yes", then rectification ratio persists upon repeated measurement of the same metal | monolayer | metal "sandwich".

\begin{tabular}{|c|c|c|c|c|c|}
\hline $\begin{array}{c}\text { Mole } \\
\text { cule }\end{array}$ & $\begin{array}{c}\text { LB or } \\
\text { LS? }\end{array}$ & $\begin{array}{c}\text { IVT/ } \\
\text { nm }\end{array}$ & RR & $\begin{array}{c}\text { Survives } \\
\text { Cycling? }\end{array}$ & Ref. \\
\hline $\mathbf{1}$ & LB & 530 & $2-27$ & No & {$[14]$} \\
\hline $\mathbf{2}$ & LS & 530 & 5 & Yes & {$[13]$} \\
\hline $\mathbf{3}$ & LS & 530 & 5 & Yes & {$[13]$} \\
\hline $\mathbf{4}$ & LB & 504 & $3-64$ & No & {$[15]$} \\
\hline $\mathbf{5}$ & LB & 480 & $8-60$ & No & {$[16]$} \\
\hline $\mathbf{6}$ & LB & 720 & 2 & No & {$[17]$} \\
\hline $\mathbf{7}$ & LS & --- & 10 & Yes & {$[18]$} \\
\hline $\mathbf{8}$ & LB & --- & $2-5$ & No & {$[19]$} \\
\hline $\mathbf{9}$ & LB & 595 & 30 & Yes & {$[19]$} \\
\hline $\mathbf{1 0}$ & LB & 1220 & 13 & No & {$[20]$} \\
\hline $\mathbf{1 1}$ & LS & --- & $6-60$ & Yes & {$[21]$} \\
\hline
\end{tabular}

The rectification measurements in the period 1997-2007 showed that: (1) a single monolayer of molecules 1 through 11 between macroscopic metal electrodes (at first $\mathrm{Al}$, then $\mathrm{Au})$ moderate rectification ratios $\mathrm{RR} \equiv$ $\mathrm{I}\left(\mathrm{V}_{\max }\right) / \mathrm{I}\left(-\mathrm{V}_{\max }\right)$ were found at $\mathrm{V}_{\max }= \pm 1 \mathrm{~V}$ or larger; (2) the RR values do vary from pad to pad, and are moderate; (3) for molecules $1,4,5,6,8$, and $10 \mathrm{RR}$ decreases progressively as the same "sandwich" or "pad" is measured repeatedly; (4) RR stays constant (no decrease) for monolayers of $\mathbf{2}$ and $\mathbf{3}$ because the molecules were transferred as an LB monolayer and immediately chemisorbed onto the Au bottom electrode, and for $\mathbf{7}$ and $\mathbf{1 0}$ because the monolayer is very spacefilling and rigid (so rigid that LS rather than LB transfer was necessary; (5) the molecules rectify in the direction opposite [3] to what was predicted by AR; (6) inelastic electron tunnelling spectroscopy of 10 at $4.2 \mathrm{~K}$ showed a broad and asymmetric signal that is characteristic of orbital-mediated tunnelling [21]: this probably means that the current really does travel through (and not around) the molecules of $\mathbf{1 0}$ [21].

\section{Newer rectifiers}

A newly designed molecule 12 (Fig. 2) does rectify electrical current (Fig. 3) [22].

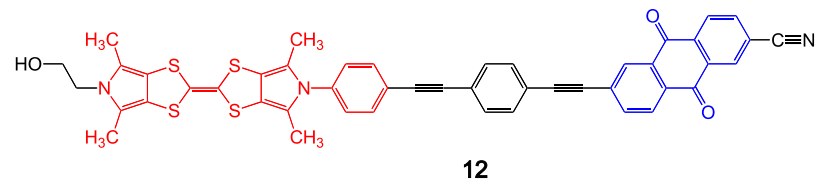

Fig. 2. Structure of a new rectifier (12) [22].

Molecule 12 chemisorbs quantitatively onto $\mathrm{Au}$, and also forms a Pockels-Langmuir monolayer at the air-water interface (presumably with the hydroxyl group closer to the water); under compression and over time molecules of $\mathbf{1 2}$ do slide past each other, quasi-reversibly, to create an aggregate multilayer (this was confirmed by atomic force microscopy) [22]. The IV curves show a rectification ratio $\mathrm{RR}$ at $\pm 1 \mathrm{~V}$ of about 80 (Fig. 3); RR increases considerably if the IV curve is studied between -2 and +2 Volts [22]. The orientation (tilt) of $\mathbf{1 2}$ in the monolayer is under study.

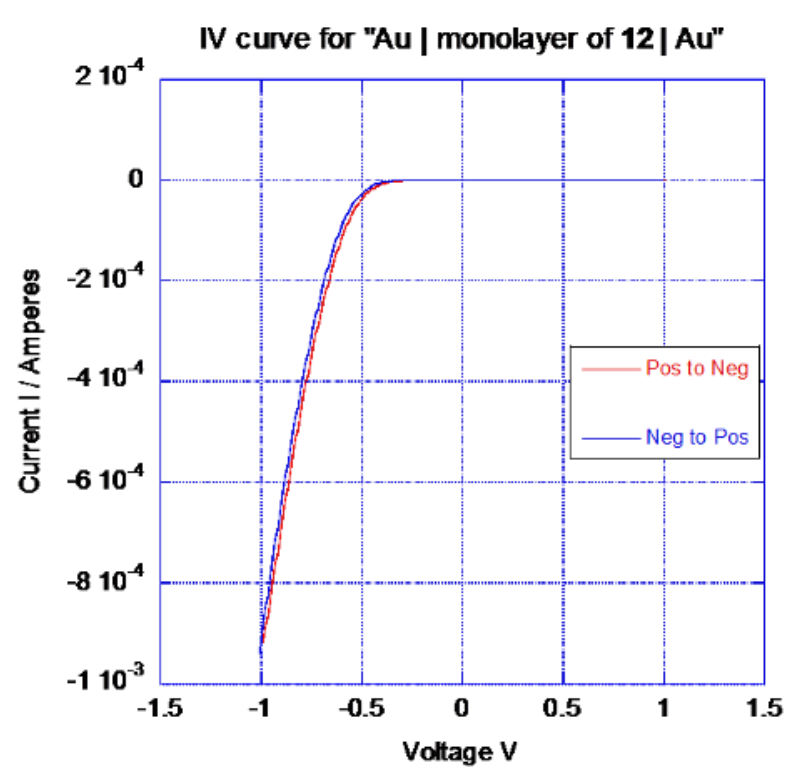

Fig. 3. IV curve for LB monolayer of new rectifier (12) [22].

\section{Unimolecular amplifier}

Theoretical calculations [11] have determined the conditions under which a three-terminal molecule, connected to three inorganic metal electrodes (Fig. 4), will be a power amplifier.

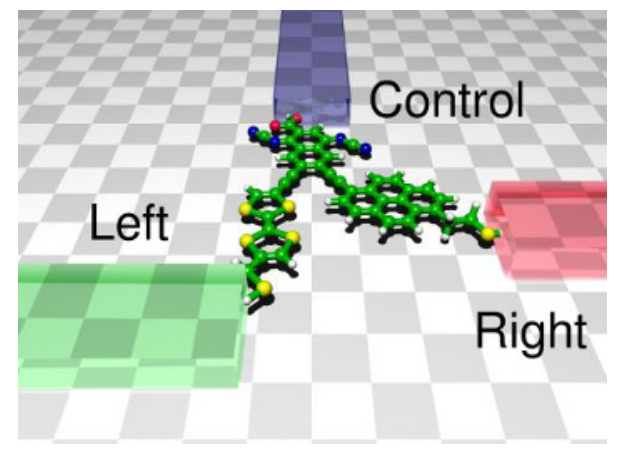

Fig. 4. Depiction of a candidate unimolecular amplifier attached to three metal electrodes (Left, Control, Right).

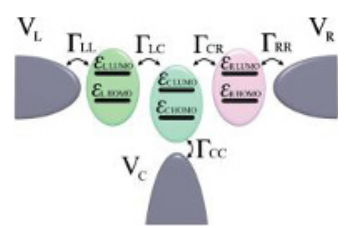

Fig. 5. The energy levels $\varepsilon_{a}$ and the coupling energies $\Gamma_{i}$ 
The algorithm was based on Ref. [23]. The energy levels of the three moieties were $\varepsilon_{a}(a=\mathrm{L}$ HOMO, L LUMO, C HOMO, C LUMO, R HOMO, or R LUMO), estimated from a DFT calculation, with an assumed Lorentzian density of states (values for $\varepsilon_{a}$ ranged between -2.5 to -5 $\mathrm{eV}) . \Gamma_{j a}$, the coupling energy to the electrodes $j(j=\mathrm{L}, \mathrm{C}$, R) and the moieties $a$, was set at $0.05 \mathrm{eV}$, but the coupling $\Gamma_{C C}$ had to be smaller: $0.001 \mathrm{eV}$. The occupation number $N_{j a}$ on each molecular level $a$ due to electrode $j$ depended on the energy $\varepsilon_{a}$ and the chemical potential $\mu_{j}$; $N_{j a}$ ranged between 0 to 2 . The chemical potential $\mu_{j 0}$ at zero bias was set to $-5.0 \mathrm{eV}$ for all $j$. The current $I_{j a}$ between electrode $j$ and level $a$ was $I_{j a}=\left(\Gamma_{i} / \hbar\right)\left(N_{j a}-N_{a}\right)$ $\left(I_{j a}>0\right.$ if electrons were entering molecule). Within the molecule, levels could modify the occupations on each other. The current $I_{a b}$ between levels $a$ and $b$ was $I_{j a}=(e$ $\left.\Gamma_{a b} / \hbar\right)\left(N_{j a}-N_{b}\right)$. At steady-state, the occupation of each level was constant, and the sum of all of the currents in and out of this level vanished. This model did not directly consider the geometry of the system. The equation for the molecular level energy was:

$$
\varepsilon_{a}=\varepsilon_{a 0}+U_{a}\left(N_{a}-N_{a 0}\right)+U_{j a}^{e e}\left(\mu_{j}-\mu_{j 0}\right)
$$

where $U_{a}$ was energy by which the molecular level $a$ changed when an electron was added to it or removed from it, while $U_{j a}^{e e}=0.03 \mathrm{eV}$ was the energy by which the molecular level $a$ changed in response to the electric field created by the voltage applied to electrode $j$. Calculations proceeded to self-consistency. The current between $\mathrm{L}$ and $\mathrm{R}$ electrodes shifted for a non-zero bias applied to the central electrode (Fig. 6): amplification was found both for when $U^{e e}{ }_{j a} \neq 0$ (field-effect), and also when $U^{e e}{ }_{j a}=0$ (bipolar junction effect).

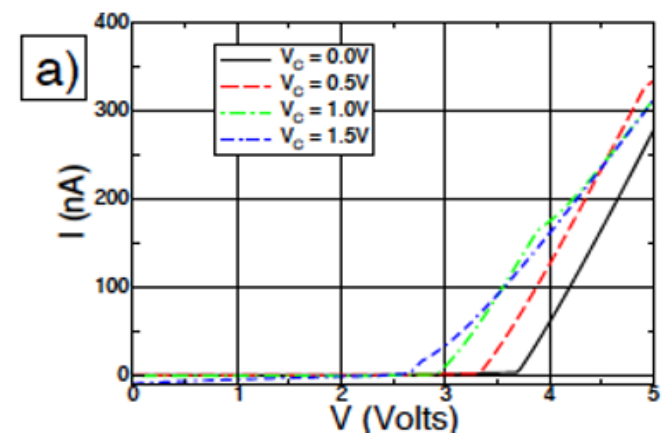

Fig. 6. Theoretical current (I)-voltage (V) plot for threeterminal molecule attached to three metal electrodes, which is displaced, as different potentials $\mathrm{V}_{\mathrm{C}}$ are applied to the center (control) electrode: this is amplification!

\section{Acknowledgments}

Profs. Woski and Cuniberti, Drs. Mahmud, Toher and Nozaki, and Mr. Johnson were instrumental in the more recent work presented here. This work was supported by the United States National Science Foundation (grant 0848306) and by the Deutsche Forschungsgemeinschaft (Mercator professorship).

\section{References}

1. A. Aviram, M. A. Ratner, Chem. Phys. Lett. 29, 277 (1974)

2. R.M. Metzger, J. Mater. Chem. 18, 4364 (2008)

3. R.M. Metzger, D.L. Mattern, Top. Curr. Chem. 313, 39 (2012)

4. G.J. Ashwell, A. Chwialkowska, Chem. Commun. 1404 (2006)

5. M. Elbing, R. Ochs, M. Koentopp, M. Fischer, C. von Hänisch, F. Weigend, E. Evers, H.B. Weber, M. Mayor, Proc. Nat. Acad. Sci. 102, 8815 (2005).

6. C.J. Muller, J. M. van Ruitenbeek, J. J. de Jongh, Physica C191, 485 (1992).

7. M.A. Reed, C. Zhou, C.J. Muller, T.P. Burgin, J.M.Tour, Science 278, 252 (1997).

8. J. Park, A.N. Pasupathy, J.I. Goldsmith, C. Chang, Y. Yaish, J.R. Petta, M. Rinkoski, J.P. Sethna, H.D. Abruña, P.L. McEuen, D.C. Ralph, Nature 417, 722 (2002).

9. G. Binnig, H. Rohrer, Ch. Gerber, E. Weibel, Physica B109-B110, 2075 (1982).

10. B. Xu, N. J. Tao, Science 301, 1221 (2003).

11. C. Toher, D. Nozaki, G. Cuniberti, R.M. Metzger, submitted.

12. A.S. Martin, J.R. Sambles, G.J. Ashwell, Phys. Rev. Lett. 70, 218 (1993).

13. A. Jaiswal, D. Rajagopal, M.V. Lakshmikantham, M.P. Cava, R.M. Metzger, Phys. Chem. Chem. Phys. 9, 4007 (2007).

14. R. M. Metzger, B. Chen, U. Höpfner, M.V. Lakshmikantham, D. Vuillaume, T. Kawai, X. Wu, H. Tachibana, T. V. Hughes, H. Sakurai, J.W. Baldwin, C. Hosch, M.P. Cava, L. Brehmer, G.J. Ashwell, J. Am. Chem. Soc. 119, 10455 (1997).

15. A. Honciuc, A. Otsuka, Y.H. Wang, S.K. McElwee, S. A. Woski, G. Saito, R.M. Metzger, J. Phys. Chem. B110, 15085 (2006).

16. J.W. Baldwin, R.R. Amaresh, I.R. Peterson, W.J. Shumate, M.P. Cava, M.A. Amiri, R. Hamilton, G.J. Ashwell, R.M. Metzger, J. Phys. Chem. B106, 12158 (2002).

17. R.M. Metzger, J.W. Baldwin, W.J. Shumate, I.R. Peterson, P. Mani, G.J. Mankey, T. Morris, G. Szulczewski, S. Bosi, M. Prato, A. Comito, Y. Rubin, J. Phys. Chem. B107, 1021 (2003).

18. A. Honciuc, A. Jaiswal, A. Gong, K. Ashworth, C.W. Spangler, I.R. Peterson, L.R. Dalton, R.M. Metzger, J. Phys. Chem. B109, 857 (2005).

19. W.J. Shumate, D.L. Mattern, A. Jaiswal, J. Burgess, D.A. Dixon, T.R. White, A. Honciuc, R.M. Metzger, J. Phys. Chem. B110, 11146 (2006).

20. W.J. Shumate, Ph.D. dissertation, University of Alabama (2005).

21. A. Honciuc, R.M. Metzger, A. Gong, C.W. Spangler, J. Am. Chem. Soc. 129, 8310 (2007).

22. I. Mahmud, M.S. Johnson, S.A. Woski, R.M. Metzger, in preparation. 
MATEC Web of Conferences

23. M. Paulsson F. Zahid, and S. Datta, in Handbook of Nanoscience, Engineering, and Technology, page 12 (CRC Press, 2003). 